\title{
Perceived Synchronization of Mulsemedia Services
}

\author{
Zhenhui Yuan, Member, IEEE, Ting Bi, Student Member, IEEE, Gheorghita Ghinea, Member, IEEE, \\ and Gabriel-Miro Muntean, Member, IEEE
}

\begin{abstract}
Multimedia synchronization involves a temporal relationship between audio and visual media components. The presentation of "in-sync" data streams is essential to achieve a natural impression, as "out-of-sync" effects are often associated with user Quality of Experience (QoE) decrease. Recently, multisensory media (mulsemedia) has been demonstrated to provide a highly immersive experience for its users. Unlike traditional multimedia, mulsemedia consists of other media types (i.e., haptic, olfaction, taste, etc.) in addition to audio and visual content. Therefore, the goal of achieving high quality mulsemedia transmission is to present no or little synchronization errors between the multiple media components. In order to achieve this ideal synchronization, there is a need for comprehensive knowledge of the synchronization requirements at the user interface. This paper presents the results of a subjective study carried out to explore the temporal boundaries within which haptic and air-flow media objects can be successfully synchronized with video media. Results show that skews between sensorial media and multimedia might still give the effect that the mulsemedia sequence is "in-sync" and provide certain constraints under which synchronization errors might be tolerated. The outcomes of the paper are used to provide recommendations for mulsemedia service providers in order for their services to be associated with acceptable user experience levels, e.g. haptic media could be presented with a delay of up to $1 \mathrm{~s}$ behind video content, while air-flow media could be released either $5 \mathrm{~s}$ ahead of or $3 \mathrm{~s}$ behind video content.
\end{abstract}

Keywords-mulsemedia, olfaction, haptic, air-flow, multimedia, synchronization, QoE

\section{INTRODUCTION}

M ULSEMEDIA, or multi-sensorial media, refers to the combination of traditional media components (i.e. text, images, and video) and objects to target other human senses (i.e. haptic, olfaction, taste, etc). Our recent research reported in [1] [2] and [3] has investigated and demonstrated the viability of mulsemedia services on achieving high user experience levels. Unlike traditional multimedia, mulsemedia aims to provide immersive communications [4] [5] [6] and enhances user Quality of Experience (QoE) [7] [8] [9]. In general, mulsemedia services may include any combination of traditional media objects such as text, graphical images, and video, as well as non-traditional media such as olfactory, haptic and skin-sensorial data. As mulsemedia is essentially

This work was supported in part by Enterprise Ireland Innovation Partnership programme.

Z. Yuan, T. Bi and G.-M. Muntean are with the Performance Engineering Laboratory, Network Innovations Centre, School of Electronic Engineering, Dublin City University, Ireland (e-mail: ting.bi2@mail.dcu.ie, \{zhenhui.yuan, gabriel.muntean\} @dcu.ie).

G. Ghinea is with the Department of Computer Science, Brunel University, UK (email: george.ghinea@brunel.ac.uk). about using these multiple media objects to communicate information to users, achieving synchronization between the component media objects that make up the mulsemedia communication systems is essential to the success of these systems. This is as information is usually conveyed by the component media objects, as well as by the relationship between the synchronized media objects [10] [11] [12] [13].

Synchronization is therefore an important issue both in mulsemedia and multimedia research. Any timing artefacts introduced by the transport network will result in time lags between the media streams. These time lags have to be corrected at the destination in order to provide an "in-sync" presentation and achieve a good user experience. Various approaches have been put forward to address synchronization issues: for instance [14] proposes a solution for Inter-Destination Media Synchronization (IDMS) using an extension of the RTP/RTCP mechanisms, yet no QoE studies are undertaken to evaluate it. A solution based on a global clock for synchronized multimedia delivery is presented and tested in [15] over two real-life scenarios (a DVB and FM network, respectively, mixed over a broadband IP network); however, no QoE results are reported. QoE results are reported in [16], which details the QoE impact of delays on watching football (soccer) on digital TV. Other multimedia synchronization studies [10] claim that audio might be played up to $120 \mathrm{~ms}$ ahead of video and, conversely, video played up to $240 \mathrm{~ms}$ ahead of audio. Both temporal skews can be accepted by users without significant impact on experience. User perceived synchronization between olfaction media and multimedia sequence is studied in [4] and [31], which show that olfaction ahead of video content is more tolerable than olfaction behind content. Therefore, synchronization is needed to ensure a temporal ordering of events in a mulsemedia system.

The lack of in-depth investigation of user perception of mulsemedia services and synchronization between the various types of media has been the major driver behind us conducting the research presented in this paper. Two types of sensorial components, haptic and air-flow, are selected and integrated into mulsemedia sequences. As human perception varies from different individuals, the user perceived $\mathrm{QoE}$ for the mulsemedia synchronization is quantified based on extensive subjective tests. Accordingly, this paper presents the results of a QoE study carried out to explore the temporal boundaries within which haptic and air-flow media objects can be successfully synchronized with video media.

This paper is organized as follows. Section II introduces the state-of-the-art related works in mulsemedia communications and media synchronization. Section III presents the synchronization related experimental setup. Section IV analyzes the subjective test results. Finally, section V concludes the paper. 


\section{RELATED WORK}

\section{A. Mulsemedia Research}

As both the means of producing and delivering rich media content have become more accessible and the exposure of individuals to such content from diverse device types and in various contexts has increased, there is a significant increase of user interest in exposure and potential adoption of novel infotainment solutions incorporating mulsemedia content. Highly popular shows involving media components which stimulate other human senses than hearing and sight which were previously only available in entertainment parks (e.g. Disney World's 4D Toy Story Mania ${ }^{1}$ or Universal Studio's 4D Shrek Ride $^{2}$ ) are moving closer to the users using the latest state of the art devices such as the Oculus Rift ${ }^{3}$ and Google Glasses ${ }^{4}$.

Aside from earlier attempts in the film and theatre industry to manually synchronise smell with certain film scenes, there is relatively little work done in the area of integrating multimedia with other media. This is unsurprising, as different types of media objects have different requirements, dictated by their very nature. In contrast to the continuous, time-sensitive nature of video and audio data, other sensorial data have other issues associated with them and raise new challenges. For instance haptic effects involve interacting with devices which some users find invasive (e.g. haptic vests) or require expert installation (e.g. haptic chairs). Using wind generating devices could affect negatively other activities which take place in the immediate proximity of the multi-sensorial content display area. Smell has a tendency to waft and diffuse through the air when released, as well as to linger. Smell generally moves slowly through the atmosphere in many directions, with certain smells taking a long time to dissipate from the air when released. Consequently, all these create a variety of usage problems for mulsemedia data, which range from directionality, intensity, context to synchronization issues. Most researched to date has been olfaction-enhanced multimedia content and results demonstrated that applications using olfactory data need to take into account any data loss issues that may be caused by the expected problems of smells taking their time to waft on to targets, diffusing in the wrong direction, interference with ambient smells and lingering of olfactory data output previously used [4] [17].

Much of the earlier work [20] that uses computer generated smell tends to focus on its use as an alternative source of output and thus creating a computing environment with optional multimodal interfaces (i.e. a computing environment that allows the user to interact with the system using different media interfaces for input and/or output). These applications use only one data medium at a time - and as such do not fit the definition of mulsemedia - and often employ olfactory data in this way as a response to some event or to signify the occurrence of some event. Work using computer generated

\footnotetext{
${ }^{1}$ Disney World's 4D Toy Story Mania, https://disneyworld.disney.go.com/ attractions/hollywood-studios/toy-story-mania/

${ }^{2}$ Universal Studio's 4D Shrek Ride, https://www.universalorlando.com/Rides/ Universal-Studios-Florida/Shrek-4-D.aspx

${ }^{3}$ Oculus Rift, http://www.oculus.com/rift/

${ }^{4}$ Google Glasses, http://www.google.com/glass/start/
}

smell combined with other media has mostly been done in the virtual reality field [21], with only one of the reported cases [12] showing any results to suggest that the addition of olfactory data may indeed be used to increase the sense of reality in virtual reality applications.

In related work [30], researchers reported on a perceptual study carried out to establish an algorithm to provide high quality inter-media stream synchronization between haptic and audio media objects in a virtual environment. Indeed, synchronization seems to be a common theme across mulsemedia research. Thus, recent work has explored synchronization of olfactory media with video content [31], whilst [40] investigated synchronization issues between different modalities.

\section{B. Olfaction Synchronization}

Studying olfactory synchronization requirements in mulsemedia systems constitutes a challenge from two very important (and different) perspectives. Firstly, olfactory data is virtual data, unlike other media streams which have the ability to be stored in some computer data form. As such, olfactory data is stored in an external device attached to a computer and its output is generated by triggering the devices' output stream, which means that theoretically it never gets transmitted over distributed systems and networks.

Consequently, synchronization measures such as delay, delay jitter and tolerable error rate do not technically apply to the olfactory data itself, rather than to the transmitted olfactory metadata and indeed they influence the generation of the remote olfaction media components by the output device. Once the output stream generation is triggered, the device releases olfaction stimuli instantaneously and continuously until another event stops it. However, smell has a tendency to linger, as well as its emission usually happening without hurried movement as it relies on atmospheric breeze to move it through the air. Smell is therefore different from the media objects multimedia applications have become accustomed to, i.e. audio, visual and traditional media (text, images, animations), whose component media streams have a transitory nature and do not experience this natural delay. In addition, the occurrence of data loss with olfactory data is something to be expected, as sensitivity to the presence of a smell tends to decrease with prolonged exposure to it, and moreover the presence of atmospheric breeze may cause the olfactory data to waft off in the wrong direction and miss the intended target. Based on these prior work outputs, we know that olfactory synchronization boundaries are less clearly defined than those associated with traditional media and the challenge is to identify them.

Secondly, in order to explore the acceptable perceptual boundaries for olfaction, existing models, such as electronic noses [41] [42], capable just of detection and identification of odours, are sorely lacking in this respect. The only alternative available to answer this question is to conduct an empirical, participant-based, study. This is indeed the approach taken by us in our work, and, in so doing, closely follows the example set by [10], who has, in empirical work, explored the human perceptual boundaries for lip and pointer synchronization, as 
well as undertaking a survey of synchronization constraints that exist between traditional media objects [11].

Perceived synchronization of olfaction-enhanced multimedia was investigated in detail in [31], which specifies a range of olfaction skews.

1) Olfaction Skews: In [31], artificial skews were introduced at intervals of 10 s ranging from -30 s to +30 s between olfactory stimuli and video content. Steps of 10 s were chosen based on human reactions to the smell. For instance, the average time for humans to sniff a scent typically ranges from $0.5 \mathrm{~s}$ to $2.5 \mathrm{~s}$ [43] and research in [12] suggests that, based on spectrum range, exposure to short varying intervals should be limited between 20 s and 30s.

2) Impact of Intermedia Skews on Perceived Olfaction: According to [31], artificially created skews, ranging in value from -30 s to +30 s between olfaction media and video media were used to achieve intermedia-skew variation. The impact of the introduced skew on user satisfaction and enjoyment of the olfaction-enhanced multimedia experience is studied. It was concluded that significant differences of participants' perception of the synchronization effect between olfactory media and video content occurred at intermedia skew values of -30 s for olfaction ahead of video content and +20 s for olfaction behind video content. In this context, beyond a skew of $+20 \mathrm{~s}$, the olfaction media no longer had the impact to improve the sense of reality of the multimedia/mulsemedia experience; it is yet to be established, however, if skews beyond -30s are still acceptable to the viewing public.

In follow-up work, synchronization issues were discussed considering a single scent and its relationship with the associated video content in [17] and multiple scents and their complex interaction in the presence of synchronised video content in [18]. Perceptual testing of olfaction-enhanced multimedia content was also reported in detail in [19].

\section{Haptic Synchronization}

Synchronization between haptic media and audio and video streams has been the target of extensive research. Accordingly, building upon earlier results reported in [34], the research in [32] describes allowable networked delays when two musical instruments (a drum and a keyboard harmonica) are played in a distributed setting; it was found that these were more stringent than in the case when one musical instrument (a tambourine) was involved [34]. In [33] results are reported on how networked delays impact QoE when two users collaboratively move an object in 3D space. Indeed, collaboration seems to be a common theme permeating haptic synchronization research, as the impact of media asynchrony - primarily between haptic and video media, but also between haptic and audio - is the focus of research reported in [36] (acceptable skews between haptic and video media in the teleoperation of a robot were reported) and [38] (where user QoE is explored for different inter-stream synchronization schemes between video and haptic media when moving an object with a haptic interface). More recently [35] also looked at acceptable skews between haptic and audio streams in a ping-pong game.
The impact of synchronization between haptic effects and multimedia content has been investigated in [35] and [36]. The authors of [35] assess the sensitivity of humans to perceive asynchrony among media signals coming from a computer application and study the haptic-to-video and haptic-to-audio skew, respectively. Statistical results of subjective test show perception rates of around $100 \mathrm{~ms}$ regardless of modality and type of device. Additionally, asynchrony was detected at $110 \mathrm{~ms}$ when a haptic event is presented before the audio and at $92 \mathrm{~ms}$ when a haptic event is presented after audio; asynchrony was detected at $87 \mathrm{~ms}$ when haptic comes before video at $125 \mathrm{~ms}$ when haptic come after video. Research reported in [36] and [37] concluded that the video communication performance was increased when haptic and video time-delay were synchronized. The authors of [38] propose a media adaptive intra-stream synchronization control scheme to enhance the QoE in audiovisual and haptic interactive IP communications.

In terms of air flow, to the best of our knowledge, there is no research work exploring its synchronization with video content in a mulsemedia set-up; however the impact of airflow direction on human perception was investigated by the research work reported in [39] with interesting results.

Concluding, we can state that most of the prior work on inter-media synchronization involving haptics has been undertaken in a computer supported collaborative work setting and has not explored in detail user QoE when either haptic or air-flow media augment video and audio streams in a viewingfor-enjoyment setting (e.g. when viewing a movie on one's laptop) - the focus of the study reported in this paper.

\section{MULSEMEDIA SYNCHRONIZATION}

Satisfactory synchronization is essential to guarantee a temporal ordering of multiple sensorial effects in a mulsemedia delivery system. Mulsemedia sequence synchronization involves relationships between multimedia sequences and sensorial effects such as olfaction, air-flow, haptic, etc, as mentioned in the previous section. In this section, the perceived synchronization of haptic and air-flow effects in mulsemedia is studied for the first time. Since human perception varies depending on individual preference, a subjective experiment is implemented with a sample of individuals.

\section{A. Participants}

48 users, including 28 males and 20 females from different backgrounds (e.g. students, staff, researchers, engineers) participated in the subjective tests. The study was promoted via institutional email and through a specially created Facebook Group. The average age of the participants was 25 years and 9 months. $95 \%$ of the participants had not been exposed to any mulsemedia subjective quality assessment before. $50 \%$ of the participants were aged below $25,40 \%$ of the subjects were aged between 26 and 30 and the remaining ones were over 30. All the participants accepted body haptic effects and were screened against anosomia and color blindness. A five euro shopping voucher was offered as incentive to each participant 


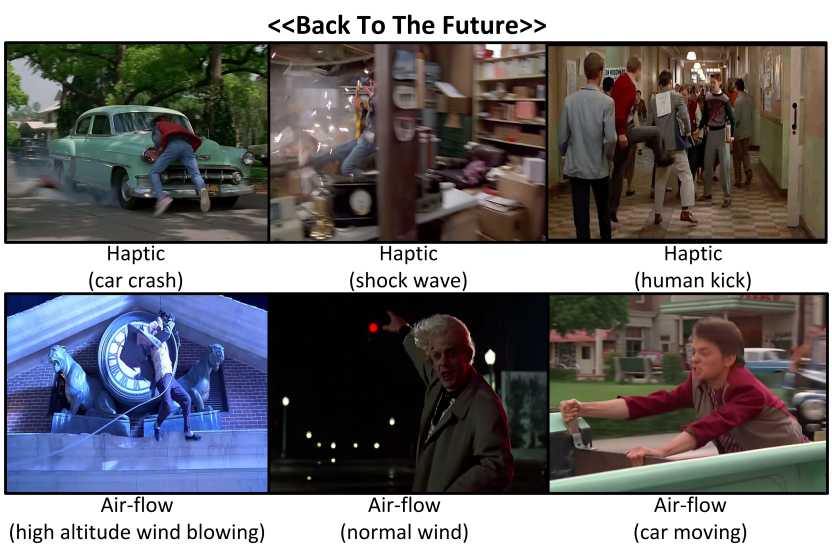

Fig. 1: Mulsemedia sequences involving multimedia components from "Back to the Future" (courtesy of Universal Studios licensing)

after the test. It took around ten minutes for each participant to complete the whole test.

\section{B. Mulsemedia Sequences}

A total of twelve 20 s video clips were selected from two movies: "Back To The Future" and "Jurassic Park". Out of the twelve video clips, six are from "Back to the Future" and the remaining six are from "Jurassic Park". 3 clips are selected from each of the two movies and are synchronized with haptic events. The remaining six clips from the two movies are synchronized with air-flow events.

All video clips had the same codec settings (i.e. a resolution of $1280 \times 720$ pixels, a frame rate of $30 \mathrm{fps}$, and a bit-rate of $2500 \mathrm{Kbps}$ ) encoded using the H.264 level 4.1 encoding. Fig. 1 illustrates the video content and lists the sensorial effects added to six (out of the twelve) sequences from "Back To The Future". Table I and Table II present the clips used, their time range relative to the overall movie they have been selected from and details of the sensorial effects employed in conjunction with each of the video clips.

The two movies were selected as they contain many scenes related to both haptic and air-flow effects, which are excellent candidates for our tests. Additionally, the team which performed this research has already used these movies in previous works and the results were very good both in terms of content diversity and subjects' interest [2]. Out of the 20s of each clip selected from the movie there are few seconds of content relevant to sensorial effects (either haptic or air-flow). The intensity of both haptic and air-flow effects was maintained constant (intensity impact has been reported in one of our previous papers [2]). Since the main purpose of this paper is to study the impact of temporal skews of sensorial effects, the strength of these effects is not considered here.

\section{Subjective Test-bed}

Unlike the case of traditional subjective testing of multimedia content, additional equipment and software were required

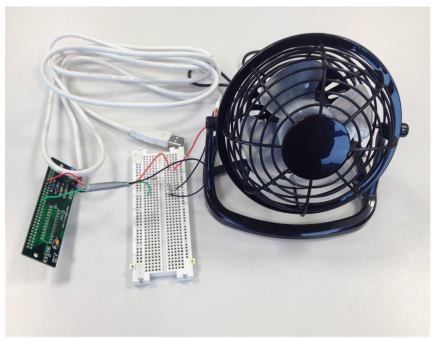

(a) USB fan

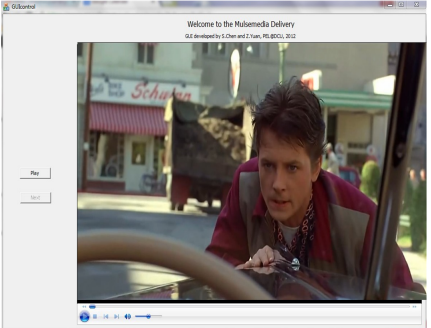

(c) player

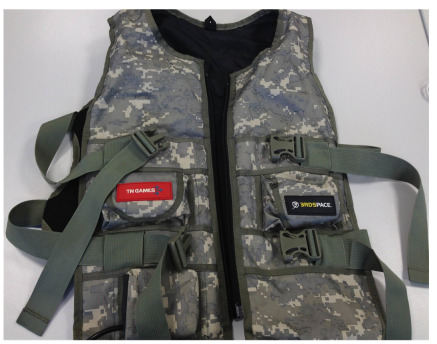

(b) haptic vest

(d) test-bed

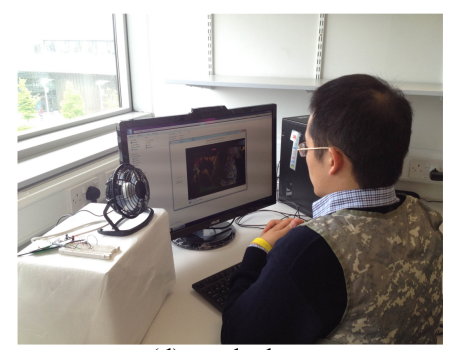

Fig. 2: The equipment and software used in the mulsemedia delivery system employed in the user tests

to present the mulsemedia sequences to the end users. A gaming vest and a USB fan were used to simulate both haptic and air-flow effects in synch with multimedia content. According to the content scenario, the haptic and air-flow effects were manually synchronized to the associated multimedia clips by setting the start and end timestamps to activate and stop the vest vibration and the USB fan operation. Figure 2 illustrates the equipment and software employed in the tests. Figure 2 (a) shows a USB fan that provided the air flow. The fan's diameter was $12 \mathrm{~cm}$ and its power was $1.4 \mathrm{~W}$. The fan's on/off state was controlled by a Visual Basic program using USBmicro ${ }^{5}$. The vibration vest in Figure 2 (b) was available from TN Games ${ }^{6}$ and supports full control (via a C++ program) of the haptic effect in terms of intensity, types, and duration. The vest simulated the precise direction and force of on-screen actions (e.g. bullet fire, explosions, environmental effects, etc.) via 8 pneumatic actuators (i.e. two on chest, two on stomach and four on the back) which generated push toward the user's body. Figure 2 (c) presents the software interface which controlled the hardware devices and played back the movie sequences for users.

The subjective test-bed shown in Figure 2 was built in a separate laboratory room in the Performance Engineering Lab at Dublin City University, Ireland. The testing environment was such setup in order to avoid any potential disturbances during the tests, obeying all the recommendations of ITU-T R.P.910 [22], ITU-T R.P.911 [23] and ITU-T R.P.913 [24]. In order for the fan to be closer to the user, but not to have any negative impact on the user assessment environment, the fan was placed on a box on top of the table at a height of $24 \mathrm{~cm}$. The distance between the fan and the user was $30 \mathrm{~cm}$.

Users wore the haptic vest sitting in front of the screen (23

\footnotetext{
${ }^{5}$ USBmicro, http://www.usbmicro.com

${ }^{6} \mathrm{TN}$ Games, http://tngames.com
} 
TABLE I: Clip Sequences from "Back To The Future" Associated with Haptic and Air Flow Effects

\begin{tabular}{|c|c|c|c|c|}
\hline Clip No. & Movie & Time Range & Effect Type & Effect Content \\
\hline 1 & Back To The Future & $41^{\prime} 55 "-42 ' 15 "$ & Haptic & Car Crash \\
\hline 2 & Back To The Future & 4'04"-4'24'” & Haptic & Shock Wave \\
\hline 3 & Back To The Future & $56 ' 25 "-56$ '45"' & Haptic & Human Kick \\
\hline 4 & Back To The Future & 1:38'38"'-1:38'58" & Air Flow & High Altitude Wind \\
\hline 5 & Back To The Future & 1:32'14"'-1:32'34"' & Air Flow & Normal Wind \\
\hline 6 & Back To The Future & 1:06'27'-1:06'47'" & Air Flow & Car Moving \\
\hline
\end{tabular}

TABLE II: Clip Sequences from "Jurassic Park" Associated with Haptic and Air Flow Effects

\begin{tabular}{|c|c|c|c|c|}
\hline Clip & Movie & Time Range & Effect Type & Effect Content \\
\hline 7 & Jurassic Park 2 & 1:27'19"'-1:27'39"' & Haptic & Beast Attack \\
\hline 8 & Jurassic Park 2 & 57’13”-57’33”' & Haptic & Car Crash \\
\hline 9 & Jurassic Park 3 & 1'46"-2'06" & Haptic & Speedboad (haptic) \\
\hline 10 & Jurassic Park 2 & $35^{\prime} 21^{\prime \prime}-35^{\prime} 41^{\prime \prime}$ & Air Flow & Car Moving (rural) \\
\hline 11 & Jurassic Park 2 & 1:54'34"'-1:54'54"' & Air Flow & Car Moving (urban) \\
\hline 12 & Jurassic Park 3 & 1'38'-1'58' & Air Flow & Speedboad (air-flow) \\
\hline
\end{tabular}

inches). Windows of the room were kept closed during the test to avoid interferences with any outside air flow (wind).

\section{Sensorial Media Synchronization Skews}

In order to generate synchronization errors, artificial skews were created using our developed software when synchronizing haptic and air-flow effects into the multimedia.

The maximum/minimum skews and the interval value were selected based on pilot tests we conducted, which indicated that air-flow skews of $[-5 \mathrm{~s}, 5 \mathrm{~s}]$ were still perceived as insync (as opposed to skews of [-3s,3s] which were enough to discriminate between in-sync and out-of-sync effects for haptic media). Thus, the lingering effect of air-flow meant that its in-sync region had more generous boundaries than its haptic counter-part. Accordingly:

1) the step size outside the $[-1 \mathrm{~s} ; 1 \mathrm{~s}]$ interval (i.e. perfect/near-perfect synchronization) for the air-flow media was larger than that of the haptic media (i.e. $2 \mathrm{~s}$ instead of 1s). However, for skews between [-1s, 1s] a granularity of $1 \mathrm{~s}$ (the minimum granularity effects could be programmed for both the air flow and the haptic media) was kept for both types of media

2) the skew range for air-flow effects was increased from $[-5 \mathrm{~s},+5 \mathrm{~s}]$ to $[-7 \mathrm{~s},+7 \mathrm{~s}]$, which increased the probability that we would be able to determine out-of-sync thresholds for air-flow media.

\section{E. Experimental Procedure}

Each of the 48 participants watched all of the 12 video clips selected from the two movies, as described in Section III. B. Six of the clips were accompanied by haptic effects, and six by air-flow effects. The allocation of participants to experimental conditions in the case of haptic effects is given in Table III (an analogous allocation was followed for air-flow effects). âĂNAccordingly, participant $\mathrm{N}$ in the experiment is allocated to Case ( $\mathrm{N}$ modulo 7) of Table III if ( $\mathrm{N}$ modulo 7$) \neq 0$, and to Case 7 otherwise. Note that $\mathrm{N}=1 . .42$ and $\mathrm{n}=1 . .7$. Consequently,

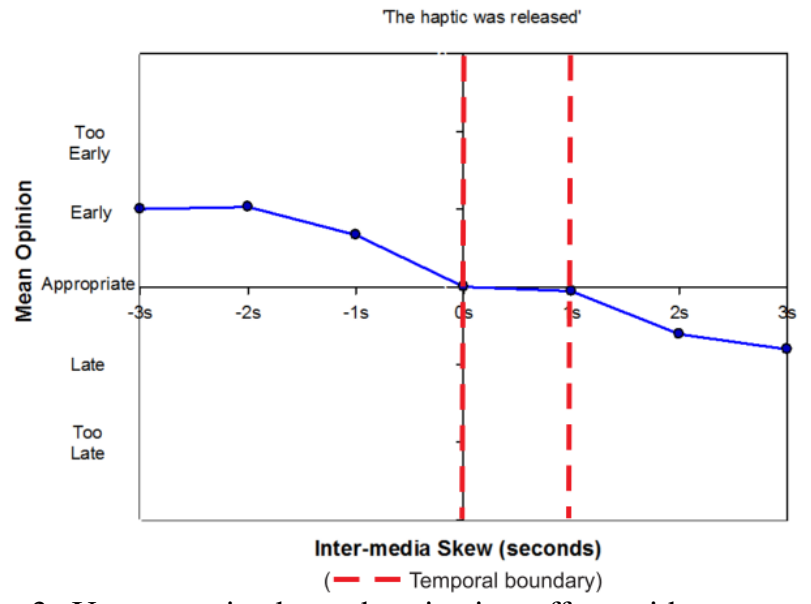

Fig. 3: User perceived synchronization effect with respect to intermedia skew between haptic and video content.

each clip was watched six times by six different participants. Moreover, in order to minimise order effects, the presentation order of the clips for each participant was randomised.

\section{Results AND ANAlysis}

This section discusses the results obtained from the subjective tests regarding the perceived synchronization of haptic and air-flow effects in a mulsemedia presentation. A test for internal consistency of our questionnaire revealed a Cronbach alpha value of 0.78 , above the recommended value of 0.7 [25], yet below the 0.9 threshold above which it has been suggested that some of its items might be redundant [26].

\section{A. Noticeability of Intermedia Skew Between Haptic/Air-flow and Video Media}

To investigate the extent to which the skews between haptic/air-flow media and video content are noticed by the users, subjective tests were conducted during which participants were asked to respond to the following statement. 
TABLE III: Intermedia Skew Values Assigned to the Haptic Effect

\begin{tabular}{|c|c|c|c|c|c|c|}
\hline Case & Clip 1 skew & Clip 2 skew & Clip 3 skew & Clip 4 skew & Clip 5 skew & Clip 6 skew \\
\hline 1 & $0 \mathrm{~s}$ & $-3 \mathrm{~s}$ & $-2 \mathrm{~s}$ & $-1 \mathrm{~s}$ & $1 \mathrm{~s}$ & $2 \mathrm{~s}$ \\
\hline 2 & $3 \mathrm{~s}$ & $0 \mathrm{~s}$ & $-3 \mathrm{~s}$ & $-2 \mathrm{~s}$ & $-1 \mathrm{~s}$ & $1 \mathrm{~s}$ \\
\hline 3 & $2 \mathrm{~s}$ & $3 \mathrm{~s}$ & $0 \mathrm{~s}$ & $-3 \mathrm{~s}$ & $-2 \mathrm{~s}$ & $-1 \mathrm{~s}$ \\
\hline 4 & $1 \mathrm{~s}$ & $2 \mathrm{~s}$ & $3 \mathrm{~s}$ & $0 \mathrm{~s}$ & $-3 \mathrm{~s}$ & $-2 \mathrm{~s}$ \\
\hline 5 & $-1 \mathrm{~s}$ & $1 \mathrm{~s}$ & $2 \mathrm{~s}$ & $3 \mathrm{~s}$ & $0 \mathrm{~s}$ & $-3 \mathrm{~s}$ \\
\hline 6 & $-2 \mathrm{~s}$ & $-1 \mathrm{~s}$ & $1 \mathrm{~s}$ & $2 \mathrm{~s}$ & $3 \mathrm{~s}$ & $0 \mathrm{~s}$ \\
\hline 7 & $-3 \mathrm{~s}$ & $-2 \mathrm{~s}$ & $-1 \mathrm{~s}$ & $1 \mathrm{~s}$ & $2 \mathrm{~s}$ & $3 \mathrm{~s}$ \\
\hline
\end{tabular}

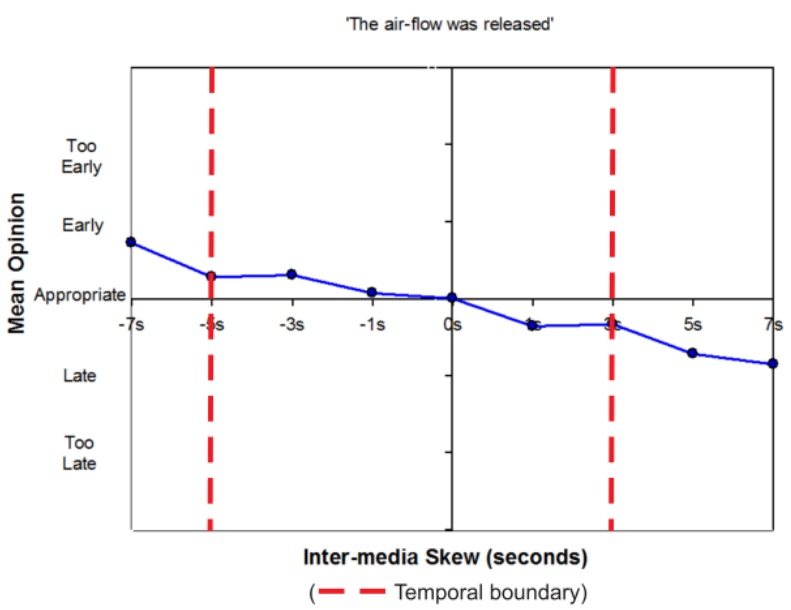

Fig. 4: Analysis of user perceived synchronization effect with respect to intermedia skew between air-flow and video content.

The haptic/air-flow effect occurred: "Too Early", "Early", "Appropriate", "Late" and "Too Late"

Fig. 3 and Fig. 4 present the perceived synchronization errors with respect to applied intermedia skew between haptic/air-flow media and video content. The left-hand side of both Fig. 3 and Fig. 4 indicate the negative skew values representing the condition where haptic and air-flow effects are ahead of the related video content, while the right-hand side represents the condition where the haptic and air-flow effects are behind the related video content.

A non-parametric Levene's test verified the equality of variances in the samples (homogeneity of variance) - $p>0.05$ [27] [28], which justified the application of the non-parametric Mann-Whitney test [29] to our data. Consequently, the nonparametric Mann-Whitney test was applied to the results, with the data from the 0s skew selected as the ground truth. If the resulting $\mathrm{P}$-value is small $(\mathrm{P}<0.05)$ then a statistically significant difference between the two samples can be accepted.

Take the haptic media for instance, the Mann-Whitney analysis shows that significant differences in participants' perception occur at an intermedia skew value lower than 0 s $(p=0.018$ at $-1 \mathrm{~s})$, i.e., haptic ahead of video content by $1 \mathrm{~s}$ or more, and at an intermedia skew value higher than $+1 \mathrm{~s}$ $(p=0.044$ at $+2 \mathrm{~s})$, i.e. haptic is behind video content by more than 1s. Take the air-flow media for instance; it is shown that significant differences in participants' perception only occur at an intermedia skew value higher than $3 \mathrm{~s}(p=0.145$ at $+3 \mathrm{~s}$ and $p=0.004$ at +5 s), i.e. air-flow is behind video content by more than $3 \mathrm{~s}$.

Consequently, the above results indicate that the temporal boundaries for synchronizing haptic and air-flow media with video content could be split in two regions, an "in-sync" region and an "out-of-sync one;

1) The "in-sync" region indicates that the presence of intermedia skew synchronization errors is acceptable to users. This region spans between $0 \mathrm{~s}$ and $+1 \mathrm{~s}$ for haptic media and between $-5 \mathrm{~s}$ and $+3 \mathrm{~s}$ for air-flow media. Therefore, haptic and air-flow media might be generated up to $0 \mathrm{~s}$ and $5 \mathrm{~s}$ ahead of video content, respectively, or delayed by a maximum of $1 \mathrm{~s}$ and $3 \mathrm{~s}$, respectively, before users start perceive the synchronization errors.

2) The "out-of-sync" areas represent the ranges in which the majority of users noticed the synchronization errors. This region spans beyond a skew of $0 \mathrm{~s}$ and $5 \mathrm{~s}$ when haptic and air-flow media are ahead of video, respectively. Additionally, the "out-of-sync" areas span beyond $1 \mathrm{~s}$ and $3 \mathrm{~s}$ when haptic and air-flow media are behind video content, respectively.

These temporal boundaries represent the synchronization requirements when haptic and air-flow media is combined together with video content. Both Fig. 3 and Fig. 4 show that although users detected the synchronization error, in general, the majority of users consider that the haptic and air-flow are generated early or late, instead of opinions such as too early or too late which reflect a more severe impression. Additionally, in terms of haptic media, the temporal boundaries show that haptic ahead of video content is more noticeable than the case when haptic is behind. On the other hand, for air-flow media, the temporal boundaries indicate that air-flow behind video content is more noticeable than that of adhead video content. This is consistent with the nature of air-flow, which lingers and drifts in the air, with the consequence that such a natural delay impacts the intermedia skews.

\section{B. Impact of Intermedia Skew on the User Perceived Experi- ence}

The impact of synchronization errors on the user perceived experience was also empirically investigated based on subjective user tests. User opinions were collected based on the responses to questions as follows.

1) Question 1: The haptic/air-flow effect enhanced the sense of relevance

2) Question 2: The haptic/air-flow effect enhanced the sense of reality 

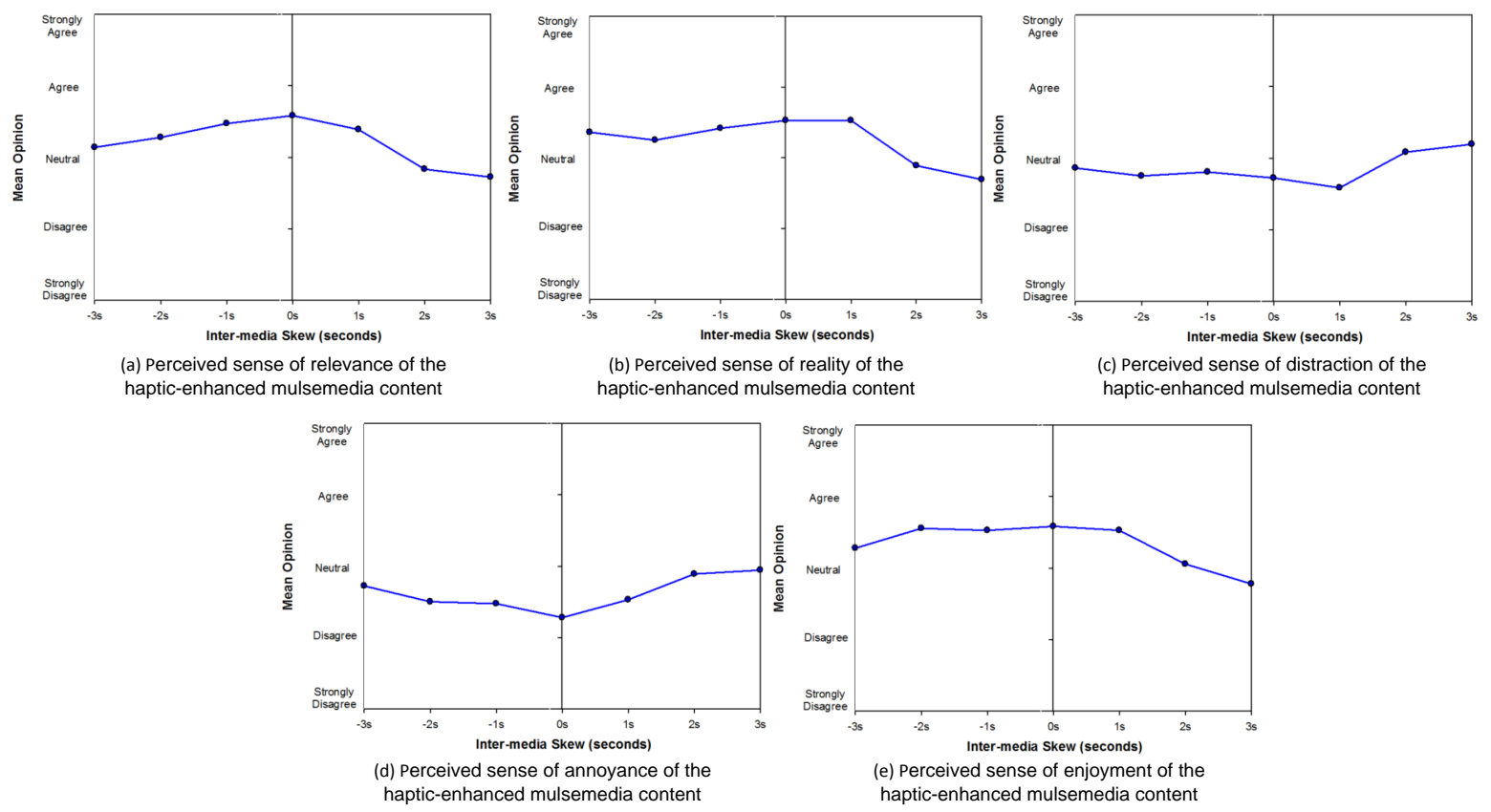

Fig. 5: Impact of intermedia skew on the user perception of the haptic enhanced mulsemedia.

3) Question 3: The haptic/air-flow effect was distracting

4) Question 4: The haptic/air-flow effect was annoying

5) Question 5: The haptic/air-flow effect enhanced the enjoyment

Each of the 48 participants stated their opinion in respect of the above statements on a 5-point Likert scale, containing the following items: "Strongly Disagree", "Disagree", "Neutral", "Agree" and "Strongly Agree". Applying a non-parametric Levine test to participant responses to each of the above statements revealed $\mathrm{p}$ values above 0.05 [27] [28], thus confirming the equality of variance assumption necessary for the application of the Mann-Whitney test.

1) Impact of Intermedia Skew on the Perceived Sense of Relevance of the Mulsemedia: Participants' agreements with question 1 are analyzed to investigate the impact of the mulsemedia clips on the sense of relevance, which refers to the level that the movie content is related to the sensorial media the user is exposed to. Fig. 5 (a) shows the sense of relevance perceived by users' in respect of haptic-enhanced video. The Mann-Whitney test indicates that with the exception of $+2 \mathrm{~s}$ and $+3 \mathrm{~s}(\mathrm{p}<0.05)$, i.e., haptic that is $2 \mathrm{~s}$ and $3 \mathrm{~s}$ behind video content, there is no significant difference in users' opinions regarding the sense of relevance of haptic enhanced video clips.

Fig. 6 (a) shows users' opinions regarding to the perceived sense of relevance of the air-flow enhanced mulsemedia. The Mann-Whitney test shows that with the exception of a skew of $+5 \mathrm{~s}(\mathrm{p}<0.05)$, i.e., air-flow that is $5 \mathrm{~s}$ behind video content, there is no significant difference in users' opinions regarding the sense of relevance of air-flow enhanced video clips. Ad- ditionally, this is consistent with the "in-sync region" of the air-flow enhanced video, identified earlier as the level at which users start to notice the synchronization error.

Furthermore, the curves in both Fig. 5 (a) and Fig. 6 (a) reflect that, in general, users have a positive bias as regards the integrated haptic and air-flow media strengthing the sense of relevance.

2) Impact of Intermedia Skew on the Perceived Sense of Reality of the Mulsemedia: Fig. 5 (b) presents users' level of (dis)agreement, in respect of the perceived sense of reality of the haptic enhanced mulsemedia. This establishes the degree of resemblance between the presented multisensorial scene and the real-life environment it represents. The Mann-Whitney analysis shows that with the exception of skews of $+2 \mathrm{~s}$ and $+3 \mathrm{~s}$ $(\mathrm{p}<0.05)$, i.e., haptic is $2 \mathrm{~s}$ and $3 \mathrm{~s}$ behind video content, there is no significant difference in users' opinions regarding the sense of reality of haptic enhanced video clips.

Fig. 6 (b) shows users' opinions in respect of the perceived sense of reality of the air-flow enhanced mulsemedia. Results from the Mann-Whitney test show that with the exception of a skew of $+5 \mathrm{~s}(\mathrm{p}<0.05)$, i.e., air-flow that is $5 \mathrm{~s}$ behind video content, there is no significant difference in users' opinions regarding the sense of reality of air-flow enhanced video clips. Additionally, this is consistent with the "in-sync region" of the air-flow enhanced video, identified earlier as the level at which users start to notice the synchronization error.

Furthermore, the curves in both Fig. 5 (b) and Fig. 6 (b) reflect that, in general, users have positive opinions in respect of the haptic and air-flow enhanced video content. 


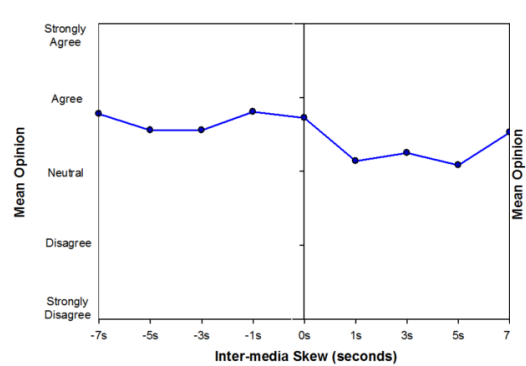

(a) Perceived sense of relevance of the air-flow-enhanced mulsemedia
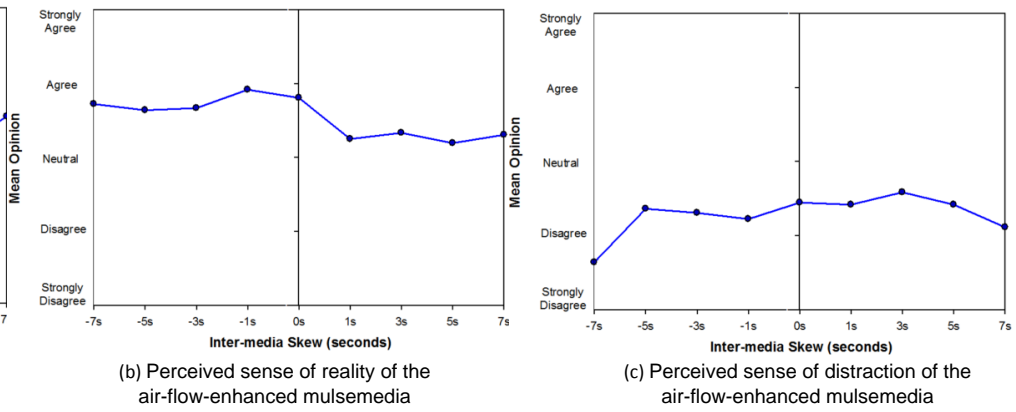

(c) Perceived sense of distraction of the
air-flow-enhanced mulsemedia

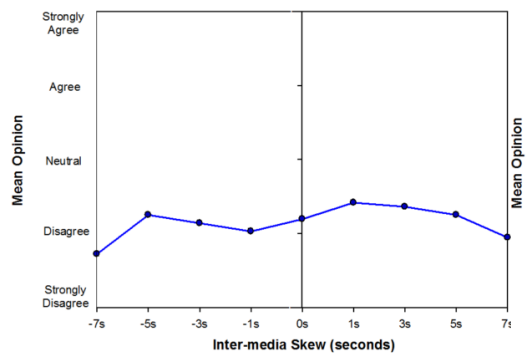

(d) Perceived sense of annoyance of the air-flow-enhanced mulsemedia

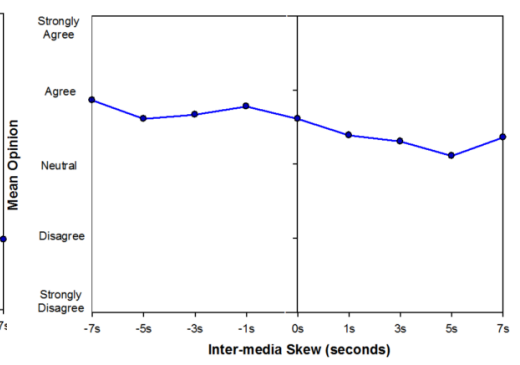

(e) Perceived sense of enjoyment of the air-flow-enhanced mulsemedia

Fig. 6: Impact of intermedia skew on the user perception of the air-flow enhanced mulsemedia.

3) Impact of Intermedia Skew on the Acceptability of the Haptic/Air-flow Enhanced Multimedia: To study the influence of intermedia skew on the acceptability of haptic and airflow integrated video content, users' responses to question 3 ("The haptic/air-flow effect was distracting") and question 4 ("The haptic/air-flow effect was annoying") are analyzed. To achieve this, the levels of distraction and annoyance perceived by users are measured when presented with "out-of-sync" areas mulsemedia.

Fig. 5 (c) shows users' opinions in terms of the perceived level of distraction for haptic enhanced video. The MannWhitney test shows that with the exception of skews of $+3 \mathrm{~s}$ $(\mathrm{p}<0.05)$, i.e., haptic is $3 \mathrm{~s}$ behind video content, there is no significant difference in users' opinions. Fig. 5 (d) shows users' opinions in terms of the perceived level of annoyance for haptic enhanced video. The Mann-Whitney test indicates that, except the skews of $+2 \mathrm{~s}$ and $+3 \mathrm{~s}(\mathrm{p}<0.05)$, i.e., haptic is $2 \mathrm{~s}$ and $3 \mathrm{~s}$ behind video content, there is no significant difference in users' opinions.

Fig. 6 (c) and Fig. 6 (d) present users' opinions regarding the perceived levels of distraction and annoyance of the air-flow enhanced video, respectively. The Mann-Whitney test shows that, between the skew range $[-7 \mathrm{~s}, 7 \mathrm{~s}]$, there is no significant difference in users' opinions.

Accordingly, our results show that there are no statistically significant differences between skews previously identified as "in-sync" and those belonging to "out-of-sync" areas in respect of user distraction or annoyance. Indeed the curves in Fig. 5 (c), Fig. 6 (c), Fig. 5 (d) and Fig. 6 (d) reflect that, in general, the majority of users had a negative bias, or were neutral, in their opinions of the level of distraction and annoyance.

4) Impact of Intermedia Skew on the Perceived Level of Enjoyment of the Mulsemedia: Lastly, the influence of intermedia skew on the perceived level of enjoyment of the mulsemedia clips is studied. Fig. 5 (e) shows users' level of (dis)agreement, in respect of the perceived level of the haptic enhanced mulsemedia. Mann-Whitney analysis shows that with the exception of skews of $+2 s$ and $+3(p<0.05)$, i.e., haptic is $2 \mathrm{~s}$ and $3 \mathrm{~s}$ behind video content, there is no significant difference in users' opinions.

Fig. 6 (e) presents users' opinions regarding the perceived levels of enjoyment of the air-flow enhanced video. The MannWhitney test reveals that, between the whole skew range $[-7 \mathrm{~s}$, $7 \mathrm{~s}$ ], there is no significant difference in users' opinions when a synchronization error is present or not.

In conclusion, the subjective results demonstrate that haptic media could be delayed by up to $1 \mathrm{~s}$ behind the video while haptic media ahead of video is not tolerable. This is also proved by other research [35] about haptic synchronization which shows that the users are more tolerant when the haptic event occurs behind the video, compared to the case when it occurs ahead of it.

\section{Conclusion and Future Work}

This paper investigates how users perceive skews in the synchronization between different sensorial components in haptic and air-flow-enhanced mulsemedia sequences. The impact of diverse inter-media skews on user quality of experience levels 
is discussed in terms of synchronization effect, sense of relevance, sense of reality, level of distraction, level of annoyance and level of enjoyment. The study has identified temporal boundaries for perfect synchronization between haptic/air-flow media and video media components, and the paper defined "in-sync regions" for both haptic (i.e., 0s to +1 s) and air-flow (i.e., $-5 \mathrm{~s}$ to $+3 \mathrm{~s}$ ) enhanced video content on this subjective test bed. These temporal boundaries are applicable to the next generation of mulsemedia solutions so that they generate an ideal immersive video experience for their end users.

Additionally, the results of this paper reveal that although users noticed the presence of some synchronization errors, their level did not significantly impact on the general perceived quality of experience of the haptic/air-flow-enhanced multimedia sequences. The majority of the users' opinions reflect positive attitudes toward the mulsemedia service in respect of the sense of relevance, the sense of reality and the level of enjoyment. Furthermore, it is concluded that haptic media could be presented with a delay by up to $1 \mathrm{~s}$ behind the video content in order to be acceptable by most of the users; in contrast air-flow media could be released either $5 \mathrm{~s}$ ahead of or $3 \mathrm{~s}$ behind the video content to achieve the acceptable level. This is because air-flow has the tendency to linger (the waft of air-flow pushed into the environment by the fan lingers long after the fan has been switched off; so even if the fan is switched off in advance, it could well be that the waft of air-flow might linger long enough, as still to be perceived in-sync with the clip content). However the converse is not true, since it is useless switching on the fan long after the corresponding moment in the video-the lingering waft of airflow and scent will be even more hopelessly out-of-sync with the video content. So it is perfectly logical that the "in-sync region" for both air-flow and olfaction are $(-m,+n)$ seconds where $m>n$.

Currently, the authors realise that cognitive bias might have played a role in our tests and that users might have just appreciated the novelty factor that mulsemedia experiences bring. In order to explore whether or not this is the case, further work has to be done, employing methods such as the repeated evaluation technique [46] or based on the methodology described in [47], specifically tailored to novel product evaluation in the context of multisensory environments.

An interesting future work direction could also consider the study of the impact of out-of-sync presentation of one or more sensorial media components such as haptic or air-flow, when the others are in-synch or out-of-synch, respectively. Moreover, building on the results of the work described in this paper, future work can explore buffer sizes needed for mulsemedia synchronization and how prioritising mulsemedia scheduling schemes can be incorporated in existing methods for inter-stream synchronization. Another avenue for future work could explore the impact of mulsemedia content type on user perception, extending the audio and video work reported in [48]. This research can also include looking at the impact of age, sex, education, working background, etc. on the temporal skews in terms of human perception of mulsemedia content, complementing the research already performed published in [49]. Not last, future work can study the manner in which different parameters related to the various sensorial components influence the user perceived quality of mulsemedia content, including intensity of the stimuli, orientation, duration, distance from the subjects, etc.

It is hoped that this paper will open-up new research avenues in the space of mulsemedia content delivery and will trigger the development of complex mulsemedia delivery systems, involving user senses of olfaction, haptic, air-flow, thermoception, and gustation, and the study of the assiciated user perceived quality, both in the presence as well as absence of interactivity.

\section{REFERENCES}

[1] Z. Yuan, G, Ghinea, G.-M. Muntean, "Beyond Multimedia Adaptation: Quality of Experience-aware Multi-sensorial Media Delivery," IEEE Transactions on Multimedia, vol.PP, no.99, pp.1,1, doi: 10.1109/TMM.2014.2371240.

[2] Z. Yuan, S. Chen, G. Ghinea, G.-M. Muntean, "User Quality of Experience of Mulsemedia Applications", ACM Transactions on Multimedia Computing Communications and Applications (TOMM), vol. 11, no. 1s, Sep. 2014

[3] Z. Yuan, G. Ghinea, and G.-M. Muntean, "Quality of Experience Study for Multiple Sensorial Media Delivery", accepted by IEEE International Wireless Communications and Mobile Computing Conference (IEEE IWCMC), Nicosia, Cyprus, Aug. 2014.

[4] O. Ademoye and G. Ghinea, "Synchronization of Olfaction-Enhanced Multimedia", 11(3), 561-565, IEEE Transactions on Multimedia, 2009.

[5] G. Ghinea and O. Ademoye, "User Perception of Media Content Association in Olfaction-Enhanced Multimedia", ACM Transactions on Multimedia Computing, Communications and Applications, 8(4), Article 52, 2012.

[6] J. G. Apostolopoulos, P.A. Chou, B. Culbertson, T. Kalker, M.D. Trott, and S. Wee, "The Road to Immersive Communication," Proceedings of the IEEE, vol.100, no.4, pp.974,990, April 2012.

[7] C. Timmerer, M. Waltl, B. Rainer, and N. Murray, "Sensory Experience: Quality of Experience Beyond Audio-Visual", in "Quality of Experience: Advanced Concepts, Applications and Methods" (S. MÃúller and A. Raake, eds.), Springer, Heidelberg, Germany, pp. 351-365, 2014

[8] S. Ud Din, D. Bulterman, "Synchronization Techniques in Distributed Multimedia Presentation", the Fourth International Conferences on Advances in Multimedia (MMEDIA), pp. 1-9, April 2012

[9] F. Boronat, M. Montagud, H. M. Stokking, and O. Niamut, "The need for inter-destination synchronization for emerging social interactive multimedia applications", IEEE Communications Magazine, 50(11), pp. 150-158, 2012

[10] R. Steinmetz, "Human perception of jitter and media synchronization", IEEE Journal on Selected Areas in Communications, vol.14, no.1, pp.6172, Jan 1996.

[11] G. Blakowski and R. Steinmetz, "A Media Synchronization Survey: Reference Model, Specification, and Case Studies", IEEE Journal on Selected Areas in Communications, vol. 14, no.1, pp. 5-35, Jan. 1996.

[12] D. A. Washburn, L. M. Jones, R. V. Satya, C. A. Bowers and A. Cortes, "Olfactory Use in Virtual Environment Training", Modelling and Simulation Magazine, vol. 2, no. 3, pp. 19-25, 2003.

[13] Z. Huang, K. Nahrstedt, and R. Steinmetz, "Evolution of temporal multimedia synchronization principles: A historical viewpoint". ACM Transactions on Multimedia Computing, Communications and Applications, 9, 1s, Article 34, 2013

[14] M. Montagud, F. Boronat, and H. Stokking, âĂİEarly event-driven (EED) RTCP feedback for rapid IDMS.âĂİ In Proceedings of the 21st ACM international conference on Multimedia (MM '13). ACM, New York, NY, USA, pp. 323-332, 2013 
[15] C. Concolato, S. Thomas, R. Bouqueau, and J. Le Feuvre, "Synchronized delivery of multimedia content over uncoordinated broadcast broadband networks", Proceedings of the 3rd Multimedia Systems Conference (MMSys '12). ACM, New York, NY, USA, pp. 227-232, 2012

[16] R. Mekuria, P. Cesar, and D. Bulterman, "Digital TV: the effect of delay when watching football", Proceedings of the 10th European Conference on Interactive TV and Video (EuroiTV), ACM, New York, USA, pp. 71-74, 2012

[17] N. Murray, Y. Qiao, B. Lee, G.-M. Muntean, "User Profile Based Perceived Olfactory and Visual Media Synchronization", ACM Transactions on Multimedia Computing Communications and Applications (TOMM), vol. 10, no. 1, Jan. 2014

[18] N. Murray, B. Lee, Y. Qiao, G.-M. Muntean, "Multiple-scent Enhanced Multimedia Synchronization", ACM Transactions on Multimedia Computing Communications and Applications (TOMM), vol. 11, no. 1s, Sept. 2014

[19] N. Murray, Y. Qiao, B. Lee, A. K. Karunakar, and G.-M. Muntean, âĂIJSubjective evaluation of olfactory and visual media synchronizationâĂ ACM Multimedia Systems Conference, Oslo, Norway, pp. 162-171, Feb 2013

[20] A. Bodnar and R. Corbett, "AROMA: Ambient awaReness through Olfaction in a Messaging Application - Does Olfactory Notification Make 'Scents'?" In Proceedings of ACM International Conference on Multimodal Interfaces (ICMI), State College, PA, USA, Oct 2004, pp.183-190.

[21] H. Q. Dinh, N. Walker, L. F. Hodges, C. Song, and A. Kobayashi, "Evaluating the importance of multi-sensory input on memory and the sense of presence in virtual environments", Proceedings of the Virtual Reality Annual International Symposium, pp. 222-228, 1999

[22] ITU-T Rec. P.910, "Subjective Video Quality Assessment Methods for Multimedia Applications", Int. Telecommun. Union, Geneva, Switzerland, Sep. 1999

[23] ITU-T Rec. P.911, "Subjective Audiovisual Quality Assessment Methods for Multimedia Applications", Int. Telecommun. Union, Geneva, Switzerland, Dec. 1998

[24] ITU-T Rec. P.913, "Methods for the Subjective Assessment of Video Quality, Audio Quality and Audiovisual Quality of Internet Video and Distribution Quality Television in Any Environment", Int. Telecommun. Union, Geneva, Switzerland, Jan. 2014

[25] L. Cronbach "Coefficient alpha and the internal structure of tests", Psychometrika, 16(3), pp. 297-334, 1951

[26] D. Streiner, "Starting at the beginning: an introduction to coefficient alpha and internal consistency", Journal of Personality Assessment. vol. 80(1), pp. 99-103, 2003.

[27] D. W. Nordstokke, and B. D. Zumbo, "A cautionary tale about Levene's tests for equality of variances", Journal of Educational Research and Policy Studies, vol. 7(1), pp. 1-14, 2007

[28] D. W. Nordstokke, B. D. Zumbo, S. L. Cairns, and D. H. Saklofske, "The operating characteristics of the nonparametric Levene test for equal variances with assessment and evaluation data", Practical Assessment, Research and Evaluation, vol. 16(5), 2, 2011

[29] D. J. Sheskin, "Handbook of parametric and nonparametric statistical procedures", 5th edition, Chapman and Hall/CRC, 2011

[30] Y. Ishibashi, T. Kanbara and S. Tasaka, "Inter-stream synchronization between haptic media and voice in collaborative virtual environments", Proceedings of the 12th Annual ACM International Conference on Multimedia, New York, NY, USA, Oct 2004, pp.604-611.

[31] G. Ghinea and O.A. Ademoye, "Perceived Synchronization of Olfactory Multimedia", IEEE Transactions on Systems, Man and Cybernetics, Part A: Systems and Humans, voo.40, no.4, pp.657-663, Jul 2010.

[32] Q. Zeng, Y. Ishibashi, N. Fukushima, S. Sugawara, and K. E. Psannis, "Influences of inter-stream synchronization errors among haptic media, sound, and video on quality of experience in networked ensenmble," in Proc. The 2nd IEEE Global Conference on Consumer Electronics (GCCE), pp. 466-470, Oct. 2013.
[33] P. Huang, Q. Zeng, and Y. Ishibashi, "QoE assessment of will transmission using haptics: Influence of network delay," in Proc. The 2nd IEEE Global Conference on Consumer Electronics (GCCE), pp. 456-460, Oct. 2013.

[34] A. Tatematsu, Y. Ishibashi, N. Fukushima and S. Sugawara, "QoE assessment in haptic media, sound and video transmission: Influences of network latency," , IEEE International Workshop in Communications Quality and Reliability (CQR), 2010, pp.1-6

[35] J. M. Silva, M. Orozco, J. Cha, A. E. Saddik, E. M. Petriu, "Human perception of haptic-to-video and haptic-to-audio skew in multimedia applications", ACM Trans. on Multimedia Computing, Communications, and Applications, vol. 9, issue 2, May 2013.

[36] H. Kim, J. H. Ryu, "A study on the effect of haptic to video timedelay on teleoperation and a comment for improving the performance", International Conference on Control, Automation and Systems, pp. 13291332, Oct. 2011.

[37] S. Kameyama and Y. Ishibashi, "Perception of synchronization errors in haptic and visual communications," in Proc. SPIE Optics East, Multimedia Systems and Applications IX, vol. 6391, no. 8, Oct. 2006.

[38] E. Isomura, S. Tasaka, and T. Nunome, "QoE enhancement in audiovisual and haptic interactive IP communications by media adaptive intrastream synchronization", Proc. TENCON, pp. 1085-1089, Nov. 2011.

[39] T. Nakano, Y. Yoshioka, and Y. Yanagida, "Effects of wind source configuration of wind displays on property of wind direction perception", International Conference on Advances in Computer-Human Interactions, pp. 365-370, 2014.

[40] E. Steinbach, S. Hirche, M. Ernst, F. Brandi, R. Chaudhari, J. Kammerl, and I. Vittorias, "Haptic Communications," Proceedings of the IEEE , vol.100, no.4, pp.937-956, Apr 2012.

[41] H. T. Nagle, R. Gutierrez-Osuna and S. S. Schiffman, "The How and Why of Electronic Noses", IEEE Spectrum, vol.35, no.9, pp.22-31, Sept 1998.

[42] L. A. Rowe and R. Jain, "ACM SIGMM retreat report on future directions in multimedia research", ACM Transactions on Multimedia Computing, Communications, and Applications, vol.1, no.1, pp.3-13, Feb 2005.

[43] D. G. Laing, "Natural sniffing gives optimum odour perception for humans", Perception, vol.12, no.2, pp.99-117, 1983.

[44] Z. Yuan and G.-M. Muntean, "A Prioritized Adaptive Scheme for Multimedia Services over IEEE 802.11 WLANs", IEEE Transactions on Network and Service Management, no.4, vol.10, pp.340 - 355, Dec. 2013.

[45] J. Xiao, T. Tillo, C. Lin, Y. Zhang and Y. Zhao, "A Real-Time Error Resilient Video Streaming Scheme Exploiting the Late- and Early-Arrival Packets," Broadcasting, IEEE Transactions on , vol.59, no.3, pp.432,444, Sept. 2013.

[46] C. C. Carbon, and H. Leder, "The repeated evaluation technique (RET). A method to capture dynamic effects of innovativeness and attractiveness", Applied Cognitive Psychology, vol. 19(5), pp. 587-601, 2005

[47] M. Jakesch, M. Zachhuber, H. Leder, M. Spingler, M., and C. C. Carbon, "Scenario-based touching: On the influence of top-down processes on tactile and visual appreciation", Research in Engineering Design, vol. 22, pp. 143-152, 2011

[48] E. J. Rodriguez, T. Nunome, and S. Tasaka, "Multidimensional QoE assessment of multi-view video and audio (MVV-A) IP Transmission: The effects of user interfaces and contents", International Conference on Advanced Information Networking and Applications Workshops, pp. 91-98, 2012

[49] N. Murray, Y. Qiao, B. Lee, G.-M. Muntean, A. K. Karunakar, "Age and gender influence on perceived olfactory and visual media synchronization", IEEE International Conference on Multimedia and Expo, pp. 1-6, July 2013 